\title{
Produção científica brasileira sobre métricas alternativas: revisão sistemática
}

\author{
Brazilian scientific production on alternative metrics: systematic literature review
}

\begin{abstract}
Márcia Regina Silva
Doutora em Educação pela Universidade Federal de São Carlos - UFSCar, Brasil. Professora do curso de Biblioteconomia e Ciência da Informação da Faculdade de Filosofia, Ciências e Letras de Rineirão Preto da Universidade de São Paulo - FFCLRP/USP. Professora titular do Programa de Pós-Graduação em Ciência da Informação da Universidade Federal de São Carlos - UFSCar, Brasil.

E-mail: marciaregina@usp.br https://orcid.org/0000-0001-5852-1026

Ednéia Silva Santos Rocha Bibliotecária da Faculdade de Direito de Ribeirão Preto da Universidade de São Paulo - USP, Brasil.

E-mail: edneia@usp.br https://orcid.org/0000-0003-1478-6828

Guilherme Moreira de Souza Bibliotecário da Luizalabs, Ribeirão Preto, Brasil E-mail: moreira183@gmail.com https://orcid.org/0000-0003-3724-6410
\end{abstract}

\begin{abstract}
Resumo
O objetivo desta pesquisa é realizar uma revisão sistemática na literatura sobre Altmetria ou métricas alternativas indexadas na base de dados Scopus, com o intuito de delinear um panorama do que tem sido discutido no âmbito nacional. A metodologia da revisão sistemática foi adotada por permitir maior controle e direcionamento das etapas que constituem o levantamento bibliográfico e a análise de dados. Ao total de registros recuperados foram estabelecidos critérios para elegibilidade e aplicados filtros para a análise documental. Após essas etapas, sínteses foram geradas a fim de representar as ideias principais dos documentos. Os resultados demonstram que os pesquisadores brasileiros com publicações indexadas na Scopus sobre Altmetria buscaram conceituar e entender os preceitos teóricos dessa abordagem quantitativa, bem como mapear o impacto social de áreas e disciplinas por meio de indicadores altmétricos. Conclui-se que pela abrangência da pesquisa, ainda não é suficiente fazer prospecções a respeito do direcionamento da Altmetria na literatura nacional. No entanto, é possível destacar que essa metodologia está sendo firmada tendo como embasamento teorias e preceitos bem consolidados pelos estudos métricos tradicionais, apoiada em ferramentas cada vez mais aprimoradas.
\end{abstract}

Palavras-chave: Altmetria. Métricas Alternativas. Revisão Sistemática.

\begin{abstract}
The aim of this research is to conduct a systematic review in the literature on Altmetry indexed in the Scopus database, in order to outline an overview of what has been discussed at the national level. The methodology of systematic review was adopted because it allows greater control and direction of the steps that constitute bibliographic survey and data analysis. The total number of records retrieved was established and criteria for document analysis were applied. After these steps, summaries were generated in order to represent the main ideas of the documents. The results show that Brazilian researchers with publications indexed in Scopus on Altmetrics sought to conceptualize and understand the theoretical precepts of this quantitative approach, besides seeking to map the social impact of areas and disciplines through altmetric indicators. It is concluded that due to the scope of the research, it is still not enough to make inquiries regarding the direction of Altmetria in the national literature. However, it is possible to highlight that this methodology is being established based on theories and precepts well consolidated by traditional metric studies, supported by increasingly improved tools.
\end{abstract}

Keywords: Atlmetrics. Alternative Metrics. Systematic Review.

InCID: R. Ci. Inf. e Doc., Ribeirão Preto, v. 11, n. 2, p. 162-184, set. 2020/fev. 2021.

DOI: 10.11606/issn.2178-2075.v11i2p162-184 


\section{Introdução}

O estudo do comportamento dos cientistas e da ciência tornou-se mais latente com as emergentes ferramentas de mensuração da ciência. No âmbito das métricas tradicionais, o impacto científico é mensurado pela citação de determinado artigo ou autor em outras publicações periódicas. Esse processo, embora reconhecido e calcado em leis e metodologias bem delineadas, é moroso e não consegue representar o interesse por uma obra específica além da academia. Segundo Maricato e Martins (2017, p. 49), as métricas tradicionais "não captam o espectro completo do 'impacto' de uma pesquisa científica e que metodologias complementares devem ser consideradas".

As metodologias complementares citadas pelos autores estão relacionadas com a Altmetria ou com métricas alternativas. A Altmetria busca abranger "a socialização de diversos resultados de pesquisas científicas, registradas em diferentes fontes sociais, nas mais diversas mídias e redes sociais, meios de comunicação e ferramentas de gerenciamento de referências" (MARICATO; MARTINS, 2017, p. 50). Também é responsável por se adequar "à multimidialidade, interatividade, hipertextualidade e à acessibilidade" (SHINTAKU et al., 2018, p. 53), associadas à aproximação de públicos acadêmicos e não acadêmicos, produzindo "uma nova composição de audiência para a ciência" (VALERIO; PINHEIRO, 2008, p. 160).

A Altmetria torna-se importante para ampliar o olhar para o impacto de uma publicação fora das fronteiras da academia, trazendo elementos para entender o impacto social de uma publicação, ou seja, a atenção e interesse da sociedade por um resultado científico. Desde 2010, quando o termo foi cunhado por Jason Priem em uma postagem no Twitter (PRIEM et al., 2010), houve um aumento das pesquisas com esse viés.

Buscando denotar o interesse pelos estudos altmétricos no âmbito da Ciência da Informação brasileira, realizou-se no mês de junho de 2020 um levantamento na Base de Dados Referencial de Artigos de Periódicos em Ciência da Informação (BRAPCI) utilizando o termo “Altmetria”. Foram recuperados 53 trabalhos distribuídos entre os anos de 2013 a 2019, cujo resultado demonstra que o tema tem despertado interesse no campo, no entanto, artigos de revisão sobre Altmetria ainda são escassos.

Nesse contexto, o objetivo desta pesquisa é realizar uma revisão sistemática da literatura nacional sobre Altmetria ou métricas alternativas com intuito de reunir as publicações e analisar sistematicamente o conteúdo desses estudos, delineando um panorama do que tem sido 
discutido na literatura sobre essa temática. O recorte para a realização desta pesquisa consiste em publicações brasileiras, sem especificação de área, indexadas na base de dados Scopus.

\section{Altmetria: vantagens e críticas}

A Altmetria tem sido utilizada como abordagem metodológica em trabalhos aplicados, que analisam o impacto social de produções científicas, instituições, pesquisadores e periódicos. Santos e Albuquerque $(2017$, p. 8$)$ ressaltaram que

[...] a análise da comunicação científica por meio das métricas e indicadores altmétricos pode ser útil para ajudar pesquisadores iniciantes interessados em melhorar seus currículos, subsidiar cientistas que desejam aumentar sua visibilidade, ou até mesmo auxiliar agências de fomento que pretendam compreender que parcela do público se interessa por uma determinada pesquisa.

Os autores ainda complementam que "a existência de práticas multiformes reativa antigas formas de sociabilidade, comunicação e informação e ao mesmo tempo desenham novos formatos de trocas e interações" (SANTOS; ALBUQUERQUE, 2017, p. 9). As mídias sociais tornaram-se o lócus que faz a ponte entre o pesquisador, sua pesquisa e a sociedade, apresentando-se como um ambiente mais democrático para o acesso aos resultados de pesquisas. A Altmetria se utiliza de ferramentas que capturam a atenção recebida pelas publicações e as transforma em scores que permitirão o monitoramento do interesse por pesquisas científicas nas redes sociais, contribuindo para a condução da comunicação na ciência (SANTOS; ALBUQUERQUE, 2017).

Os profissionais da informação e bibliotecários atuantes em unidades de informação utilizam indicadores altmétricos na esfera do marketing institucional, no estudo de usuário e na gestão de coleções, sendo possível entender, por exemplo, quais são as plataformas da web preferidas de seus usuários/leitores/visitantes/clientes e quais os conteúdos recebem mais atenção (SOUZA, 2015). Conforme complementa Borrego (2014, p. 353, tradução nossa), os "dados altmétricos podem ser úteis como novos metadados que ajudam o leitor na busca de informações e o bibliotecário na identificação dos hábitos e necessidades de informação de seus usuários". Adie (2014, tradução nossa) ainda reforça que a Altmetria pode ser utilizada como ferramenta de conscientização e gestão de pesquisas.

Grande parte da produção acadêmica permanece sem citações nas bases de dados, fato que pode significar que o artigo não despertou interesse ou mesmo que possui erros no processo de citação ou de indexação, pois para que a contagem das citações seja realizada efetivamente, 
as indicações das referências bibliográficas devem estar padronizadas. Ao contrário das fontes bibliométricas que representam documentos estáticos, grande parte das fontes de informação altmétricas é dinâmica, ou seja, pode sofrer alterações ou exclusões, fazendo com que a precisão, consistência e reprodutibilidade sejam as principais limitações dessas métricas. Além disso, "a maior parte dos dados está nas mãos de empresas com fins lucrativos, o que restringe a transparência" (BASAVARAJ et al., 2017, p. 20, tradução nossa). Por possuir dados voláteis e estar inserida em um contexto comercial e político, a Altmetria está sujeita à manipulação artificial do número de curtidas, de seguidores, retweets, compartilhamentos, citações e/ou comentários, ao utilizar perfis falsos ou mesmo bots (robôs) (SOUZA, 2015).

Vogl et al. (2018, p. 1267, tradução nossa) ponderam que "as métricas alternativas ainda estão à beira da validação e ainda precisam provar que são de alguma utilidade para a comunidade científica. Mais notavelmente, deve-se ter cuidado ao vinculá-los ao prestígio de um pesquisador individual". Adie (2014, p. 351, tradução nossa) confirma esse ponto de vista ao dizer que "a disponibilidade e a confiabilidade dos dados são críticas [...] a natureza dos dados altmétricos é muito mais transitória e difícil de capturar, pelo menos de uma maneira que permita uma auditoria clara e rastreamento dos dados".

As críticas são consistentes, no entanto, as ferramentas altmétricas estão em constante aprimoramento e buscam minimizar algumas questões como a manipulação artificial dos dados, por exemplo. Outras questões como a volatilidade dos dados podem ser relativizadas, já que os indicadores altmétricos medem o interesse instantâneo por uma publicação, ou seja, seu propósito justamente é esse: denotar o impacto social em um momento específico, diferentemente das métricas tradicionais que constroem índices de citação após dois anos da publicação. Basavaraj et al. (2017, p. 16, tradução nossa), relatam que no âmbito das métricas tradicionais "o fator de impacto expressa as citações por outro periódico científico" e a "pontuação altmétrica produz uma resposta imediata sobre um artigo individual e reflete o interesse público (bem como científico) nos resultados da pesquisa".

Borrego (2014, p. 356, tradução nossa) enfatiza que "devemos entender melhor a cobertura de fontes de dados altmétricos e padronizar os procedimentos de coleta de dados para garantir a consistência na construção de indicadores".

Vogler et al. (2017, p. 174, tradução nossa) explicam que há a "necessidade de fontes altmétricas, como o Altmetric.com, expandirem o escopo limitado do identificador único DOI, se quiserem atender às expectativas de incorporar um espectro mais amplo de produção 
científica do que os procedimentos bibliométricos tradicionais". Entretanto, para realizar tal ação é necessária a criação de normas e procedimentos para padronização dessa ciência, assim como explicado por Carpenter et al. (2016, p. 87, tradução nossa) ao retratarem as discussões dos membros da NISO que elaboraram cinco temas principais a serem abordados pelos cientistas, sendo

(a) desenvolvimento de definições específicas para métricas alternativas e sua avaliação; (b) descrição dos principais usos e como se aplicam aos diferentes grupos e partes interessadas; (c) definição de métricas e metodologias de cálculo apropriadas; (d) promoção e facilitação do uso de identificadores persistentes nas comunicações acadêmicas e (e) desenvolvimento de estratégias para melhoria da qualidade dos dados.

Além da comunidade acadêmica continuar os estudos sobre a Altmetria, os desenvolvedores de softwares e aplicativos bem como os cientistas de dados também necessitam de atenção e de investimento no tema, de maneira a aprimorar os métodos de detecção e reparação de atividade fraudulenta junto ao desenvolvimento de normas e padrões da área, assim como apontado por Konkiel (2016) e Barros (2015).

Embora não seja o interesse do presente estudo comparar as métricas tradicionais e as alternativas - mesmo porque possuem variáveis e interesses diferentes -, ressalta-se que a expectativa sobre os indicadores altmétricos apoia-se tanto na disponibilidade das emergentes ferramentas altmétricas, que fazem a medição instantânea da atenção dada a uma publicação científica em redes sociais, como também nas críticas já enraizadas quanto às métricas tradicionais. 


\section{Metodologia}

Esta pesquisa é uma revisão sistemática, que de acordo com Sampaio e Mancini (2007, p. 84) consiste em "métodos sistemáticos usados para evitar viés e possibilitar uma análise mais objetiva dos resultados, facilitando uma síntese conclusiva sobre determinada intervenção", além de ser uma forma de pesquisa que tem como "fonte de dados a literatura sobre determinado tema" (SAMPAIO; MANCINI, 2007, p. 84).

Cerrao, Jesus e Castro (2018, p. 106) caracterizam a revisão sistemática "como um método de revisão da literatura que garante à pesquisa maior confiabilidade e reprodutibilidade". Os autores afirmam que essa tipologia de revisão necessita de algumas fases: "(1) planejamento que engloba revisão bibliográfica para habituação com o tema estudado; (2) condução que equivale ao processo de busca nas bases de dados e validação dos critérios de estratégia para inclusão e exclusão e (3) extração de dados", que consiste no agrupamento de todos os dados coletados, seguidos pela leitura, análise e síntese das informações. Para atingir efetivamente a fase 2 (condução), é importante seguir algumas etapas, demonstradas na Figura 1.

Figura 1 - Etapas de uma Revisão Sistemática

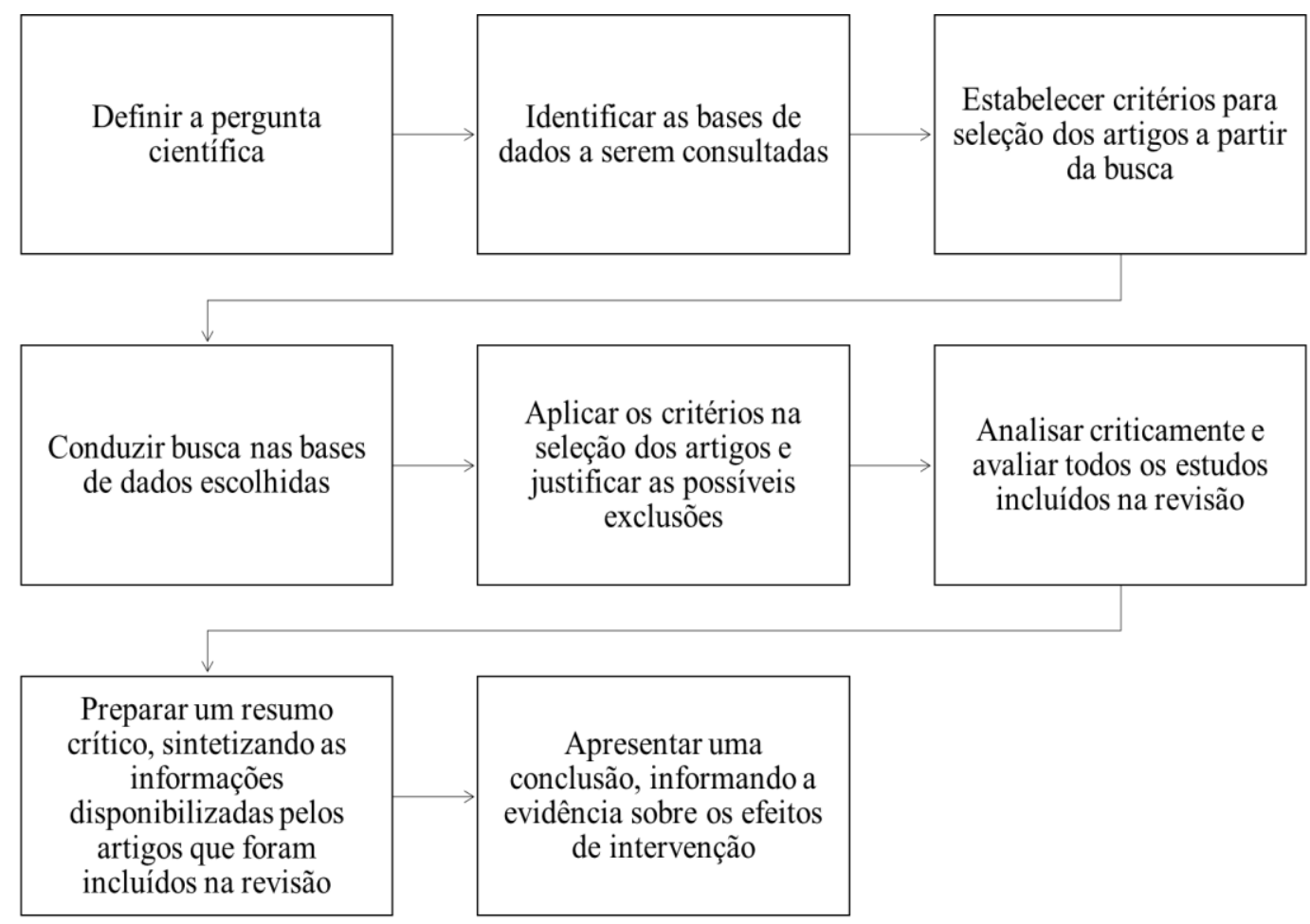

Fonte: Sampaio e Mancini (2007, p. 4)

Seguindo as etapas da Figura 1, definiu-se a seguinte pergunta científica: "como se caracterizam as pesquisas brasileiras sobre Altmetria, indexadas na base de dados Scopus?". 
No Quadro 1 e na Figura 2 apresentam-se os critérios da pesquisa seguindo o modelo apresentado por Basavaraj et al. (2017, tradução nossa).

Quadro 1 - Critérios para definição e inclusão do corpus da pesquisa

\begin{tabular}{|c|c|}
\hline Etapas da Revisão Sistemática & Delimitações da Pesquisa \\
\hline $\begin{array}{l}\text { Fontes de informação para a coleta de } \\
\text { dados }\end{array}$ & Base de Dados Scopus \\
\hline Critérios de Elegibilidade & Sem delimitação de data \\
\hline Critérios de Inclusão & Publicações brasileiras \\
\hline Critérios de Exclusão & $\begin{array}{l}\text { Publicações que fizeram menção aos conceitos, } \\
\text { mas que não tratavam da temática }\end{array}$ \\
\hline Critério de Busca & article title, abstracts, Keywords \\
\hline Termos de Busca & $\begin{array}{l}\text { altmetric OR altmetrics OR alternative metrics } \\
\text { OR alternative metric OR altimetria OR } \\
\text { altmetria OR métricas alternativas }\end{array}$ \\
\hline $\begin{array}{l}\text { Outras fontes que podem ser utilizadas } \\
\text { para embasar a pesquisa }\end{array}$ & Literatura da área de métricas da informação \\
\hline $\begin{array}{l}\text { Critério de exclusão após leitura técnica } \\
\text { dos documentos }\end{array}$ & $\begin{array}{l}\text { Trabalhos que não possuem abordagem teórica } \\
\text { ou metodológica sobre Altmetria ou métricas } \\
\text { alternativas }\end{array}$ \\
\hline
\end{tabular}

Fonte: Elaborado pelos Autores (2020).

A escolha da base Scopus justifica-se por constituir atualmente no maior banco de dados de publicações revisadas por pares, disponibilizando resumos e citações de artigos científicos, livros, anais de congressos e outras publicações. Importantes autores dos estudos métricos da ciência como Grácio e Oliveira (2012) ressaltam a relevância da escolha da base Scopus, uma vez que é considerada na atualidade como a maior base de dados multidisciplinar para se acessar a produção científica nacional e internacional. Jacso (2005) destaca ainda que a Scopus, o Google Scholar e a Web of Science são as maiores e mais importantes fontes de pesquisa multidisciplinares.

A caracterização das publicações brasileiras sobre Altmetria indexadas na Scopus pode denotar o posicionamento da comunidade científica diante das questões relacionadas às métricas alternativas. Na Scopus realizou-se a busca nos campos article title, abstracts, keywords, pois são campos que podem representar os assuntos principais dos documentos, sem delimitação temporal, valendo-se da seguinte estratégia de busca: (altmetric OR altmetrics OR alternative metrics $O R$ alternative metric $O R$ altimetria $O R$ Altmetria $O R$ métricas alternativas). Na Figura 2, apresenta-se o número de trabalhos recuperados de acordo com os critérios estabelecidos para inclusão na Revisão Sistemática. 
Figura 2 - Revisão Sistemática e Elegibilidade

\begin{tabular}{|c|c|}
\hline \multicolumn{2}{|c|}{ Mecanismo de busca (SCOPUS) } \\
\hline \multirow{2}{*}{\multicolumn{2}{|c|}{$\begin{array}{l}\text { Busca literária } \\
\text { Termos (altmetrics OR alternative metrics) }\end{array}$}} \\
\hline & \\
\hline \multicolumn{2}{|c|}{ Total de documentos em busca eletrônica (1504) } \\
\hline \multicolumn{2}{|c|}{$\begin{array}{c}\text { Documentos avaliados para eligibilidade, por meio da aplicação } \\
\text { de filtro Brazil (55) }\end{array}$} \\
\hline $\begin{array}{l}\text { Documentos incluídos no } \\
\text { estudo (25) }\end{array}$ & $\begin{array}{l}\text { Documentos que não } \\
\text { atenderam aos critérios de } \\
\text { inclusão (30) }\end{array}$ \\
\hline
\end{tabular}

Fonte: elaborado pelos autores com base no modelo de Basavaraj (2017)

Conforme a Figura 2, recuperaram-se 1.504 documentos. Para se refinar por publicações de autores brasileiros, delimitou-se no filtro de país de afiliação (AFFILCOUNTRY) o termo "Brazill, resultando assim em 55 documentos. Além dos critérios estabelecidos, a aplicação de dois filtros tornou-se necessária para incluir ou excluir publicações, sendo o primeiro relacionado à conferência de palavras-chave e leitura técnica e o segundo atrelado à leitura integral dos textos. Nesse processo, após a aplicação dos filtros nos 55 documentos, 30 documentos foram excluídos por não abordarem a Altmetria como temática principal. O corpus total da pesquisa consistiu-se, portanto, em 25 documentos.

A representação gráfica dos indicadores foi desenvolvida no programa Microsoft Excel e padronizados os dados para melhor visualização dos resultados. Especificamente, a análise Clusters das palavras-chave que representam as publicações brasileiras sobre Altmetria indexadas na Scopus foi utilizado o software VOSviewer para construir e visualizar os mapas bibliométricos. Para tanto, exportaram-se da base de dados os registros em formato Research Information System (RIS) para que o software pudesse realizar então os relacionamentos entre os descritores das publicações. 


\section{Revisão Sistemática sobre Métricas Alternativas}

Os 25 documentos (artigos e proceedings) que fizeram parte da pesquisa foram lidos na íntegra. A maioria das pesquisas pode ser categorizada como estudos aplicados $(n=21)$, os quais por vieses diferentes, buscaram levantar a reputação acadêmica e social principalmente de áreas e disciplinas. Quatro (04) trabalhos focaram a discussão teórica, por meio de levantamento bibliográfico sobre o tema Altmetria.

Pesquisadores da área de Ciência da Informação foram responsáveis por 68\% (n=17) das publicações. A proximidade da Ciência da Informação com os métodos de avaliação da produção científica pode ser evidenciada tanto na literatura como também nas discussões postas nos eventos da área, como no Encontro Nacional de Pesquisa em Ciência da Informação (ENANCIB) que possui um Grupo de Trabalho (GT) específico para as discussões relacionadas a essa área do conhecimento: o GT Produção e Comunicação da Informação em Ciência, Tecnologia \& Inovação. Outro evento muito significativo é o Encontro Brasileiro de Bibliometria e Cientometria que está na sua sétima edição. Alvarez e Caregnato (2017) por meio de uma pesquisa bibliográfica buscaram compreender a importância da Ciência da Informação para a avaliação da produção do conhecimento científico. Segundo os autores, a relevância dessa área deve-se principalmente pelas definições de leis e teorias que são utilizadas nas análises métricas para o entendimento do comportamento e da estrutura da produção científica. Conforme apontam Maia e Caregnato (2004), o desenvolvimento dos estudos bibliométricos está relacionado ao desenvolvimento da Ciência da Informação.

Nesse sentido, pelo aspecto histórico e até mesmo pelo delineamento das pesquisas no âmbito da comunicação científica empreendidos na Ciência da Informação, não surpreende que a maioria dos trabalhos seja desenvolvido por pesquisadores dessa área. Entre os trabalhos levantados, o de Gouveia (2019) aborda justamente a Ciência da Informação nos estudos sobre Altmetria. O autor faz um estudo bibliométrico sobre Altmetria tendo como parâmetro a produção científica nacional registrada na plataforma Lattes. A pesquisa revela que o campo é dominado por pesquisadores da grande área de Ciências Sociais Aplicadas, destacando-se a Ciência da Informação e, em menor grau, a área da Comunicação. Segundo Gouveia (2019, p. 1), "as abordagens vêm evoluindo, percorrendo um caminho que vai desde aquelas mais teóricas até as práticas, avaliativas, críticas e as contextualizações institucionais e regionais”.

Barros (2015) fez um estudo teórico com intuito de apresentar a Altmetria e analisar sua aplicação para avaliar o impacto acadêmico. Já Vanti e Sanz-Casado (2016) realizaram revisão 
teórica a respeito das métricas de informação com ênfase na Altmetria e concluíram que a Altmetria pode ser uma métrica alternativa complementar às tradicionais, pois permite que a contagem de citações ou menções à produção acadêmica mundial seja mais justa e igualitária, dando lugar a uma ciência também mais democrática.

Embora os estudos apontem a importância da Altmetria para o monitoramento da atenção social de publicações científicas e a função democrática desse método de avaliação, Barata (2019) em um estudo de abordagem teórica aponta limitações da Altmetria para a ciência praticada na América Latina. Segundo a autora,

a altmetria não tem sido capaz de retratar a atenção que artigos científicos brasileiros recebem nas redes sociais, apesar de haver esforços por parte das revistas em divulgação científica e do relativo engajamento social que conseguem mobilizar. É preciso ampliar a coleta de dados no Facebook, em português e espanhol, além de amplificar as coleções de blogs e veículos jornalísticos na região (BARATA, 2019, on-line).

A Altmetria é também discutida no contexto da ciência aberta por alguns autores dessa revisão. Oliveira (2019) por meio do levantamento bibliográfico aborda essa temática no editorial de um número especial da Revista Transinformação. Appel, Albagli e Maciel (2017) discutiram como a Ciência Aberta tem impactado o universo dos periódicos acadêmicos de acesso aberto. Os autores apontam características e práticas emergentes vinculadas aos processos de submissão, avaliação, revisão, edição, publicação, distribuição, acesso e uso. Os resultados demonstraram aspectos importantes das publicações examinadas, como os tipos de licenças utilizadas, as políticas de acesso a dados de pesquisa, formatos de publicação, cobrança e impacto das métricas alternativas de avaliação. Cintra, Furnival e Milanez (2018) analisaram as possíveis vantagens que o acesso aberto pode trazer para a área da Ciência da Informação. Para a análise, foram utilizados indicadores de citação e escores altmétricos de 16 periódicos da CI com maiores fatores de impacto no Journal Citation Report (JCR). Na pesquisa, foram utilizadas como fonte para coleta de dados a Web of Science (WoS), Google Scholar e Altmetric. Os resultados indicaram que o acesso aberto aumenta o número de citações e de menções nas mídias sociais para amostra do estudo. Os autores pontuaram que o aumento é maior quando os autores pagam taxas de processamento para garantir a disponibilidade imediata do acesso aberto ao trabalho. Concluíram também que embora esse tipo de acesso possa aumentar o número de citação de um artigo, a qualidade do artigo é que verdadeiramente impulsiona a maioria das citações. 
É válido destacar que 51 autores, dentre autorias individuais e coautorias, foram responsáveis pelos 25 trabalhos dessa revisão. Dois autores da área de Ciência da Informação destacam-se nesse quantitativo: Ronaldo Araújo da Universidade Federal de Alagoas (UFAL), autor ou coautor em cinco trabalhos, e João Maricato da Universidade de Brasília (UnB), que publicou três trabalhos em coautoria.

Araújo (2015) discorreu teoricamente sobre os principais indicadores do marketing científico digital e situou a Altmetria como método para coleta e análise de tais indicadores, demonstrando que a criação e a análise de indicadores altmétricos sejam pensadas nesse âmbito. Também em 2015, em coautoria, Araújo et al. realizaram a análise altmétrica da Revista Brasileira Data Grama Zero por meio do uso de APIs do Facebook e Twitter. Os resultados demonstraram que uso de APIs pode representar uma resposta para os periódicos que não possuem DOI, já que a pesquisa por URLs é aplicável independentemente de o periódico ter ou não o referido identificador, sugerindo que há um nível de cobertura muito maior do que aquele mostrado na ferramenta Altmetric.com. Araujo, Murakami e Prado (2017) refletiram sobre as interações do Facebook enquanto elemento de atenção on-line e fonte de dados altmétricos. Os autores concluíram a partir de uma análise empírica dos artigos de periódicos da Ciência da Informação indexados no Repertório da Produção Periódica Brasileira de Ciência da Informação (RPPBCI) que o Facebook é uma fonte importante para o estudo altmétrico.

Mais recentemente, Pontes e Araújo (2019) analisaram o impacto que os artigos científicos dedicados ao tema Museum Mundan promovem na web social. Por meio da abordagem altmétrica, os autores pontuaram que a "viabilização de novos estudos sobre o museu para atrair novas atenções exponencialmente quantificáveis, com a finalidade de despertar atenção e financiamentos à instituição, e, por conseguinte colaborar com o reconhecimento contemporâneo dos visionários" (PONTES; ARAÚJO, 2019, p. 199). Também em 2019, Batista, Anastácio e Araújo analisaram por meio da Altmetria o impacto da produção em políticas públicas. Os autores comprovaram a eficácia dessa métrica para analisar o impacto da produção dessa temática, vista a importância das redes sociais para circulação de material político e como nova esfera pública para discussão de temas da hora e de relevância social.

Já Maricato e Martins (2017) refletiram sobre o embasamento teórico da Altmetria e concluíram que a área possui grande potencial, no entanto necessita de mais reflexões, principalmente no que se refere aos fundamentos teóricos. Além disso, os autores apontam algumas incertezas em relação à institucionalização da área, bem como controvérsias sobre as 
possibilidades dos indicadores medirem o impacto da produção acadêmica. Também em 2018, Maricato e Vilan Filho analisaram aspectos, características e potencialidades para a mensuração do impacto social nas mídias sociais. Os autores utilizaram a base de dados Scielo para a coleta de artigos e a ferramenta Altmetric.com para análise dos perfis de indivíduos no Facebook e Twitter que agiram ou reagiram aos artigos analisados. Os perfis foram categorizados em impacto social e acadêmico. Os resultados indicaram que o impacto medido usando a Altmetria reproduz consideravelmente a relação cientista-cientista, assim como a bibliometria e a cientometria. No entanto, os autores concluíram que a Altmetria tem potencial para medir além do impacto acadêmico.

Maricato em colaboração com Lima analisaram aspectos sobre os impactos proporcionados pela Altmetria a partir da análise de dados do Facebook e Twitter como fontes de análise. Os autores concluíram que o Impacto Acadêmico é mais elevado que o Impacto Social e que o Facebook possui um número maior de usuários que se enquadram no perfil de Impacto Social do que o Twitter (MARICATO; LIMA, 2017).

Alguns estudos focaram a análise altmétrica de Periódicos Científicos. Oliveira et al. (2019) refletiram sobre o impacto social da ciência ao investigarem a presença, circulação e atenção on-line das revistas da área de Comunicação e Informação. Os resultados demonstraram que a área de Comunicação e Informação tem baixa presença tanto nas plataformas Dimensions e Altmetric quanto nos próprios sites de redes sociais. Ademais, a circulação da produção científica refere-se mais à

divulgação de pesquisadores individuais do que perfis institucionais dos periódicos, com baixo engajamento do público não acadêmico. No entanto, tem havido aumento progressivo desta presença e atenção online, apontando para um crescimento do uso de sites de redes sociais para a divulgação da produção científica como estratégia de comunicação institucional dos periódicos (OLIVEIRA, 2019, p. 285).

Silva Filho e Vanz (2019) aferiram o impacto das mídias sociais Mendeley, Facebook e Twitter sobre a visibilidade e o número de citações recebidas pelos artigos publicados na Revista Gaúcha de Enfermagem. Os resultados revelaram que trabalhos não citados tiveram impacto altmétrico por meio de ações em outras mídias sociais, acadêmicas ou não. As autoras sugerem que a adoção de métodos qualitativos, como análise de redes sociais e análise de conteúdo, pode trazer outras perspectivas para um melhor entendimento do fenômeno. Além da Ciência da Informação, outra área que se destaca entre os trabalhos é a saúde. Vieira e Sanna $(2013$, p. 2) ressaltam que 
[...] a aderência de pesquisadores aos estudos bibliométricas aplicados na área da saúde não é apenas uma tendência internacional. A Biblioteca Virtual em Saúde (BVS) também apresenta expressivos números de pesquisadores nacionais que empreenderam a análise da literatura científica, ainda com grande variação de frequências ao longo dos anos, mas de forma crescente, da década de 2000 em diante.

A utilização da abordagem bibliométrica na área de saúde como pontuado por Vieira e Sanna (2013) também pode ser observada nesta pesquisa, uma vez que 20\% ( $n=5)$ dos trabalhos apresentam estudos aplicados nessa área. Araujo et al. (2020) analisaram fatores associados aos scores altmétricos para trabalhos de revisão relacionados à dor lombar e concluíram que o fator de impacto, como o número de citações dos artigos levantados, está associado às pontuações altmétricas. Araujo et al. (2018) também utilizando uma amostra de ensaios clínicos sobre dor lombar, analisaram fatores potenciais dessas publicações associados aos scores altmétricos. A conclusão dos autores denotou uma preocupação dos pesquisadores no que tange à publicação, que deve incluir a seleção de periódicos com alto fator de impacto para a submissão e a utilização de títulos declarativos e interrogativos.

Ainda na área de saúde, dois trabalhos utilizaram o protótipo REALM (Researcher Evaluation Alternative Metrics), que tem como finalidade identificar a reputação acadêmica e social de pesquisadores e suas pesquisas a partir de dados altmétricos. Maia, Lenzi e Oliveira (2019), responsáveis pelo desenvolvimento desse protótipo, explicaram que o sistema foi concebido de forma a facilitar as tarefas de encontrar especialistas em domínios específicos, coletar material bibliográfico, melhorar a divulgação científica entre outras funcionalidades. Os autores aplicaram o REALM no cenário da febre Chikungunya e observaram que as análises realizadas pelo sistema são consistentes. Em outra pesquisa, Maia et al. (2019) implementam o REALM e a aplicaram no cenário do Zika vírus, evidenciando nomes e estudos mais importantes relacionados à doença em questão e a conscientização pública foi analisada por meio de Altmetria.

Também tendo como foco o Zika vírus, Maia e Oliveira (2017) investigaram o impacto dos principais pesquisadores em Zika e a repercussão de suas pesquisas na população, sendo identificados os autores em redes de coautoria e por meio de índices altmétricos. A repercussão dos pesquisadores foi analisada nas mídias sociais e os autores do estudo em questão concluíram que o alto número de compartilhamentos em alguns casos indica que a pesquisa teve grande repercussão nas redes sociais. Além disso, os pesquisadores de maior visibilidade estão conduzindo avanços significativos relacionados ao vírus, como também são líderes em renomadas instituições de pesquisa. 
Fora do contexto da área da Ciência da Informação e da saúde, destacam-se: o trabalho de Benchimol, Cerqueira e Papi (2014) que analisaram o periódico História, Ciências, Saúde Manguinhos sob o ponto de vista de sua visibilidade e inserção nas redes sociais e; o trabalho de Nabout et al. (2018) que avaliaram fatores associados ao impacto social de pesquisas na área de Ecologia. Os autores investigaram a influência relativa do tempo de citação, colaboração e indicadores baseados em citação em nove plataformas (Twitter, Facebook, Mendeley, Google Plus, Sina Weibo, Blog, Notícias, Vídeos e Research High-light Plataform) e concluíram que as métricas derivadas de diferentes plataformas on-line são concordantes com as métricas baseadas em citações, indicando que o conhecimento científico está se tornando disponível para diferentes públicos de maneira semelhante. Fora do espectro das áreas mencionadas anteriormente, estudo de Lucas et al. (2017), categorizado na área da Tecnologia, discorre sobre o uso de indicadores para a avaliação de atividades científicas e tecnológicas, tendo os Institutos de Ciências, Tecnologia e Inovação como focos do estudo.

A produção brasileira sobre Altmetria indexada na base de dados Scopus demonstra que o tema necessita de maior investigação, posto que os autores das pesquisas levantadas ainda colocam muitas ponderações tanto nos aspectos teóricos (MARICATO; MARTINS; 2018) como também em relação à abrangência dos indicadores altmétricos (BARATA, 2019). No entanto, os resultados das pesquisas demonstram a potencialidade da Altmetria para a mensuração além do impacto acadêmico (MARICATO; VILAN FILHO, 2018).

Na Figura 3 são apresentadas as relações entre as palavras-chave dos artigos que compõem esta revisão, representadas por quatro clusters que denotam com clareza o conceito e contexto da Altmetria. No cluster central, ilustrado pela cor verde, observa-se que o termo Altmetrics conecta-se a todos os grupos. Observou-se nos clusters que o descritor altmetrics relaciona-se principalmente aos termos: bibliometrics; open access; scientific communication; social media; social network analysis; social networking (online) e social networks.

As definições de Altmetria apresentadas neste trabalho podem ser sintetizadas em: métricas alternativas no sentido de complementariedade às métricas tradicionais como a Bibliometria; métrica social que representa a repercussão de um resultado científico nas redes sociais e acadêmicas; métrica inserida no contexto dos periódicos de acesso aberto, garantindo o rastreamento das pesquisas por meio de identificadores eletrônico e; método quantitativo para a mensuração da atenção on-line, ou impacto social, da produção científica que circula em redes 
sociais. Tais definições podem ser observadas nas conexões entre as palavras-chave representadas na Figura 3.

Figura 3 - Clusters das palavras-chave que representam as publicações brasileiras sobre Altmetria indexadas na Scopus

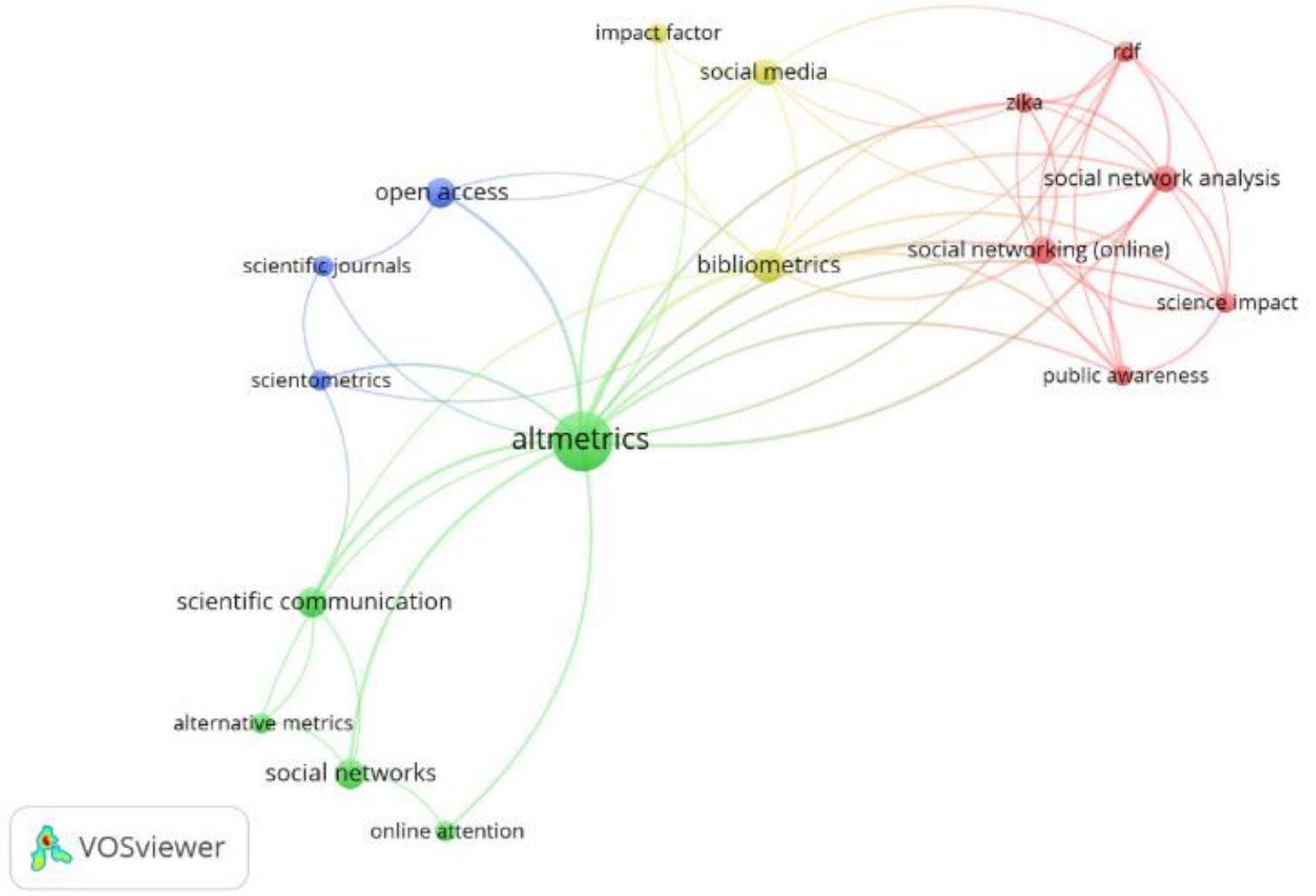

Fonte: Elaborado pelos Autores (2020)

Além do destaque ao conteúdo dos trabalhos que foram levantados, serão apresentados alguns indicadores dessa produção, com início pelo ano de publicação dos trabalhos. De acordo com o Gráfico 1, observa-se que o ano de 2019 teve o maior número de publicações. Embora a produção analisada não seja densa e desconsiderando-se o ano de 2020, ainda corrente, há uma ascendência no número de publicações. A publicação do ano de 2014 embora não desenvolva o termo Altmetria foi considerada por abordar a visibilidade e inserção social de um periódico. 
Gráfico 1 - Distribuição anual do número de publicações brasileiras sobre Altmetria (Scopus)

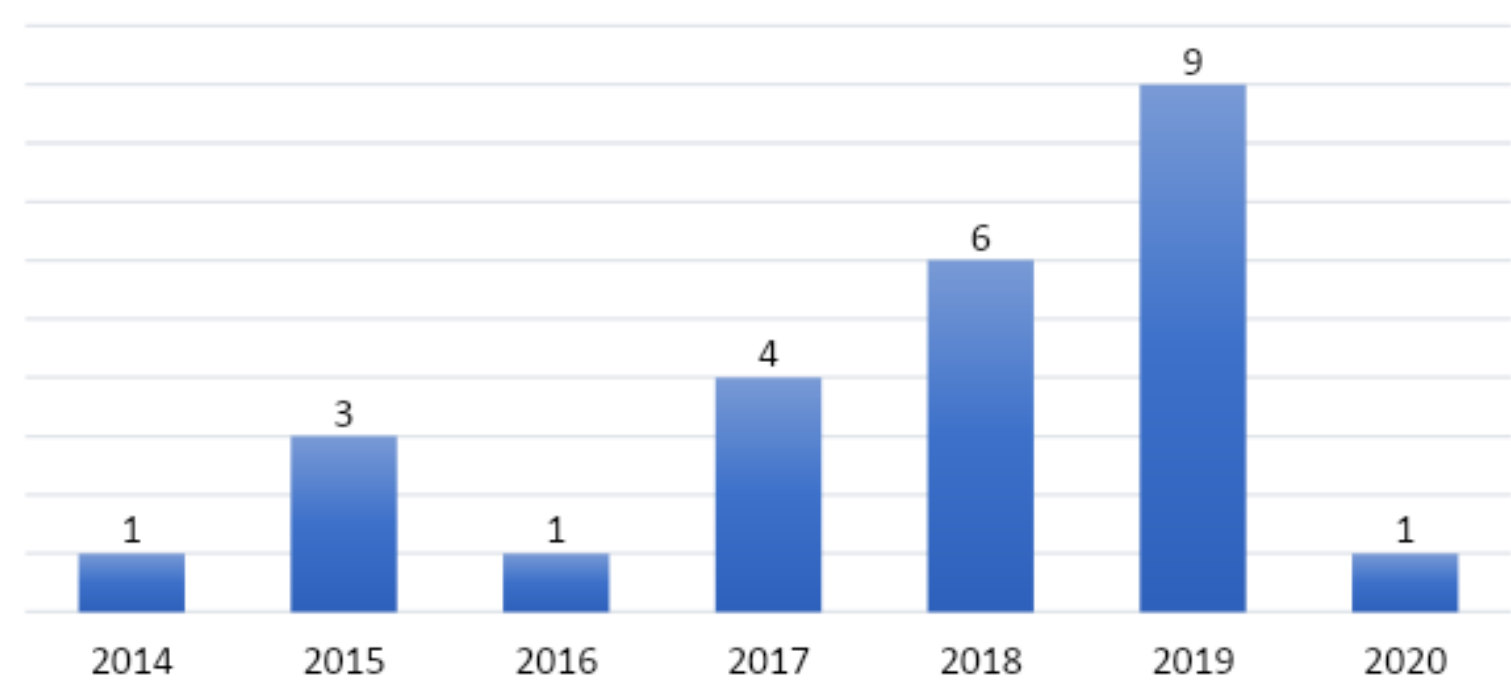

Fonte: Elaborado pelos Autores (2020)

Um indicador interessante a se destacar é a fonte de publicação dos trabalhos (Tabela 1). Os periódicos com mais de uma publicação são nacionais: Transinformação editada pela Pontifícia Universidade Católica de Campinas, Ciência da Informação do IBICT e a Perspectivas em Ciência da Informação editada pela Universidade Federal de Minas Gerais. Cinco publicações são trabalhos de eventos internacionais e três foram publicados em periódicos de outras áreas.

Tabela 1 - Fontes das publicações brasileiras sobre Altmetria (Scopus)

\begin{tabular}{|l|c|}
\hline Fontes das publicações & Contagem \\
\hline Transinformação & 5 \\
\hline Ciência da Informação & 2 \\
\hline Perspectivas em Ciência da Informação & 1 \\
\hline Revista Digital de Biblioteconomia e Ciência da Informação & 1 \\
\hline Educação e Pesquisa & 1 \\
\hline Information Research & 1 \\
\hline $\begin{array}{l}\text { Proceedings of the International Conference on WWW/Internet 2017 and Applied } \\
\text { Computing 2017 }\end{array}$ & 1 \\
\hline Brazilian Journal of Physical Therapy & 1 \\
\hline Proceedings of the 25th Brazillian Symposium on Multimedia and the Web, WebMedia 2019 & 1 \\
\hline Information Services and Use & 1 \\
\hline $\begin{array}{l}\text { Proceedings of ISSI 2015 Istanbul: 15th International Society of Scientometrics and } \\
\text { Informetrics Conference }\end{array}$ & 1 \\
\hline Informação e Sociedade & 1 \\
\hline $\begin{array}{l}\text { Proceedings - 2019 IEEE/WIC/ACM International Conference on Web Intelligence, WI } \\
\text { 2019 }\end{array}$ & 1 \\
\hline Scientometrics & \\
\hline $\begin{array}{l}\text { WebMedia 2017 - Proceedings of the 23rd Brazillian Symposium on Multimedia and the } \\
\text { Web }\end{array}$ & \\
\hline
\end{tabular}

InCID: R. Ci. Inf. e Doc., Ribeirão Preto, v. 11, n. 2, p. 162-184, set. 2020/fev. 2021. 


\begin{tabular}{|l|c|}
\hline Biblios & 1 \\
\hline Journal of Medical Internet Research & 1 \\
\hline Investigacion Bibliotecologica & 1 \\
\hline Total Geral & $\mathbf{2 5}$ \\
\hline
\end{tabular}

Fonte: Elaborado pelos Autores (2020)

As fontes de publicações apresentadas na Tabela 1 denotam que os pesquisadores brasileiros têm buscado divulgar suas pesquisas sobre Altmetria nos principais periódicos nacionais e internacionais, principalmente da Ciência da Informação, assim como em eventos internacionais.

Sobre a repercussão dos trabalhos, observou-se na base de dados Scopus a soma das citações dos trabalhos. Na Tabela 2, são apresentados os trabalhos que tiveram uma ou mais citações.

Tabela 2 - Soma de citações na base de dados Scopus dos trabalhos sobre Altmetria

\begin{tabular}{|c|c|}
\hline Título dos Trabalhos & $\begin{array}{c}\text { Soma } \\
\text { das } \\
\text { citações }\end{array}$ \\
\hline Does the global South have altmetrics? Analyzing a Brazilian LIS journal & 8 \\
\hline $\begin{array}{l}\text { Do traditional scientometric indicators predict social media activity on scientific } \\
\text { knowledge? An analysis of the ecological literature }\end{array}$ & 6 \\
\hline $\begin{array}{l}\text { The performance impact of the Brazilian information science journals on facebook: An } \\
\text { altmetric study }\end{array}$ & 6 \\
\hline Altmetrics: Alternative metrics of scientific impact based on social media & 5 \\
\hline $\begin{array}{l}\text { Impact of low back pain clinical trials measured by the altmetric score: Cross-sectional } \\
\text { study }\end{array}$ & 5 \\
\hline $\begin{array}{l}\text { Altmetrics: Social media metrics for a more democratic science [Altmetria: A métrica social } \\
\text { a serviço de uma ciência mais democrática] }\end{array}$ & 4 \\
\hline $\begin{array}{l}\text { Scientific digital marketing and altmetrics for academic journals: From visibility to } \\
\text { engagement }\end{array}$ & 4 \\
\hline $\begin{array}{l}\text { Challenges to the publishers of humanities in scientific journalism and social networks: } \\
\text { Reflections and experiences }\end{array}$ & 4 \\
\hline $\begin{array}{l}\text { The potential for altmetrics to measure other types of impact in scientific production: } \\
\text { Academic and social impact dynamics in social media and networks }\end{array}$ & 3 \\
\hline $\begin{array}{l}\text { The impact of open access citation and social media on leading top information science } \\
\text { journals }\end{array}$ & 2 \\
\hline Altmetrics impact: Aspects observed with profiles analyzes on Facebook and Twitter & 2 \\
\hline $\begin{array}{l}\text { Investigation of research impacts on the zika virus. An approach focusing on social network } \\
\text { analysis and altmetrics }\end{array}$ & 2 \\
\hline $\begin{array}{l}\text { Altmetrics: complexities, challenges and new forms of measuring and comprehending } \\
\text { scientific communication in the sociali }\end{array}$ & 1 \\
\hline Open scientific journals: Emerging practices and approaches & 1 \\
\hline $\begin{array}{l}\text { ReALM: An altmetrics-based framework to map science impacts on society. A case study } \\
\text { on Zika research }\end{array}$ & 1 \\
\hline
\end{tabular}

Fonte: Elaborado pelos Autores (2020)

O trabalho mais citado é de Fausto, Araujo, Murakami e Lara com oito citações. Tratase de um trabalho publicado no proceedings do International Conference on Scientometrics \& Informetrics de 2015. O trabalho de Nabout et al. (2018) teve seis citações e foi publicado no 
periódico Scientometrics ao passo que o trabalho de Araújo, Murakami e Prado (2018), embora também com seis citações, foi publicado na Revista Digital Biblioteconomia e Ciência da Informação. Observa-se que Ronaldo Ferreira Araújo é coautor nos dois trabalhos mais citados, despontando-se como pesquisador da temática no Brasil.

\section{Considerações finais}

Entende-se a Altmetria como método de avaliação científica, complementar aos métodos tradicionais, como a Bibliometria e a Cientometria. Os indicadores altmétricos, para representar a atenção social de publicações científicas que circulam em redes sociais, devem ser contextualizados, uma vez que tanto as ferramentas como as limitações apontadas neste trabalho influenciam a abrangência das métricas alternativas.

A utilização da Revisão Sistemática demonstrou-se eficaz neste estudo e a sistemática utilizada para o levantamento e categorização dos dados permitiu maior confiabilidade dos resultados apresentados. Os resultados denotaram que os pesquisadores brasileiros com publicações sobre Altmetria indexadas na Scopus buscaram conceituar e entender os preceitos teóricos dessa abordagem quantitativa, além de buscarem mapear o impacto social de áreas e disciplinas por meio de indicadores altmétricos.

Pela abrangência da pesquisa, ainda não é suficiente fazer prospecções a respeito do direcionamento da Altmetria na literatura nacional. No entanto, é possível destacar que essa metodologia está sendo firmada tendo como embasamento teorias e preceitos bem consolidados pelos estudos métricos tradicionais, apoiada por uma série de ferramentas cada vez mais aprimoradas. 


\section{Referências}

ADIE, E. Taking the alternative mainstream. El Profesional de la Información, León, v. 23, n. 4, p. 349-351, 2014. Disponível em:

https://recyt.fecyt.es/index.php/EPI/article/view/epi.2014.jul.01/16957. Acesso em: 12 fev. 2019.

ALVAREZ, G. R.; CAREGNATO, S. E. A Ciência da informação e sua contribuição para a avaliação do conhecimento científico. BIBLIOS: Revista do Instituto de Ciências Humanas e da Informação, Rio Grande, v. 31, n. 1, p. 9-26, 2017. Disponível em:

https://www.brapci.inf.br/index.php/res/v/22515. Acesso em: 27 jul. 2020.

APPEL, A. L.; ALBAGLI, S.; MACIEL, M. L. Open scientific journals: emerging practices and approaches. Information Services \& Use, Amsterdam, v. 37, n. 4, p. 475-488, 2017 https://content.iospress.com/articles/information-services-and-use/isu862. Acesso em: $27 \mathrm{jul}$. 2020 .

ARAUJO, A. C. et al. Impact of low back pain clinical trials measured by the altmetric score: Cross-sectional study. Journal of Medical Internet Research, Baltimore, v. 20, n. 4, 2018. Disponível em: https://www.jmir.org/2018/4/e86/. Acesso em: 27 jul. 2020.

ARAUJO, A.C. et al. The impact of low back pain systematic reviews and clinical practice guidelines measured by the Altmetric score: cross-sectional study. Brazilian Journal of Physical Therapy, São Carlos, 2020. Disponível em: http://www.rbf-bjpt.org.br/en-pdfS1413355519306641. Acesso em: 27 jul. 2020.

ARAÚJO, R. F. et al. Does the global South have altmetrics? analyzing a Brazilian LIS journal. In: INTERNATIONAL CONFERENCE ON SCIENTOMETRICS \&

INFORMETRICS, 15., 2015, Istambul. Proceedings... Turkey, 2015. p. 111-112. Disponível em: https://www.scopus.com/record/display.uri?eid=2-s2.0-

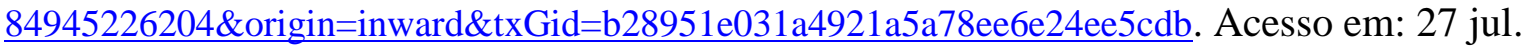
2020.

ARAÚJO, R. F. Marketing científico digital e métricas alternativas para periódicos: da visibilidade ao engajamento. Perspectivas em Ciência da Informação, Belo Horizonte, v. 20, n. 3, p. 67-84, 2015. Disponível em: https://www.Scielo.br/pdf/pci/v20n3/1413-9936-pci20-03-00067.pdf. Acesso em: 27 jul. 2020.

ARAÚJO, R. F.; MURAKAMI, T. R. M.; PRADO, J. M. K. A repercussão de artigos de periódicos brasileiros de ciência da informação no Facebook: um estudo altmétrico. In: ABEC MEETING, 2017, Curitiba. Anais... Campinas: Revista Digital de Biblioteconomia e Ciência da Informação, 2018. p. 365-379. Disponível em:

https://periodicos.sbu.unicamp.br/ojs/index.php/rdbci/article/view/8650461/pdf. Acesso em: 27 jul. 2020.

BARATA, G. Por métricas alternativas mais relevantes para a América Latina.

Transinformação, Campinas, v. 31, 2019. Disponível em: https://www.Scielo.br/Scielo.php?script=sci_arttext\&pid=S0103-37862019000100313. Acesso em: 27 jul. 2020. 
BARROS, M. Altmetrics: métricas alternativas de impacto científico com base em redes sociais. Perspectivas em Ciência da Informação, Belo Horizonte, v. 20, n. 2, p. 19-37, 2015. Disponível em: http://www.Scielo.br/pdf/pci/v20n2/1413-9936-pci-20-02-00019.pdf. Acesso em: 12 fev. 2019.

BASAVARAJ, P. et al. Altmetrics: a collated adjunct beyond citations for scholarly impact: a systematic review. Journal of Clinical and Diagnostic Research, India, v. 11, n. 6. p. 1620, 2017. Disponível em: https://jcdr.net/article_fulltext.asp?issn=0973709x \&year=2017\&volume=11\&issue=6\&page= ZE16\&issn=0973-709x\&id=10078. Acesso em: 04 out. 2018.

BATISTA, M. C. L.; ANASTÁCIO, L. A.; ARAÚJO, R. F. Menções à produção científica sobre políticas públicas de pesquisadores da UFMG na web social: um breve estudo altmétrico. Ciência da Informação, Brasília, v. 48, n. 3, p. 191-198, 2019. Disponível em: http://revista.ibict.br/ciinf/article/view/4871. Acesso em: 27 jul. 2020.

BENCHIMOL, J. L.; CERQUEIRA, R. C.; PAPI, C. Challenges to the publishers of humanities in scientific journalism and social networks: reflections and experiences. Educação e Pesquisa, São Paulo, v. 40, n. 2, p. 347-364, 2014. Disponível em: https://www.Scielo.br/pdf/ep/v40n2/en_v40n2a04.pdf. Acesso em: 27 jul. 2020.

BORREGO, Á. Altmétricas para la evalución de la investigación y el análisis de necesidades de información. El Profesional de la Información, León, v. 23, n. 4, p. 352-357, 2014. Disponível em: https://recyt.fecyt.es/index.php/EPI/article/view/epi.2014.jul.02/16958. Acesso em: 12 fev. 2019.

CARPENTER, T. A. et al. Developing standards for emerging forms of assessment: the NISO altmetrics initiative. The Serials Librarian, Estados Unidos, v. 70, n. 1-4, p. 260-265, 2016. Disponível em: https://www.tandfonline.com/doi/full/10.1080/0361526X.2016.1157737. Acesso em: 12 fev. 2019.

CERRAO, N. G.; JESUS, A. F.; CASTRO, F. F. O método de revisão sistemática da literatura (RS) na área da Ciência da Informação no Brasil: análise de dados de pesquisa. Informação \& Tecnologia, Marília/João Pessoa, v. 5, n. 1, p. 105-116, 2018. Disponível em: http://hdl.handle.net/20.500.11959/brapci/110396. Acesso em: 22 abr. 2019.

CINTRA, P. R.; FURNIVAL, A. C.; MILANEZ, D. H. The impact of open access citation and social media on leading top information science journals. Investigación Bibliotecológica, Ciudad de México, v. 32, n. 77, p. 117-132, 2018. Disponível em: http://revib.unam.mx/ib/index.php/ib/article/view/57874. Acesso em: 27 jul. 2020.

GOUVEIA, F. C. Estudos altmétricos no Brasil: uma análise a partir dos currículos da Plataforma Lattes-CNPq. Transinformação, Campinas, v. 31, 2019. Disponível em: https://www.Scielo.br/Scielo.php?script=sci_arttext\&pid=S0103-37862019000100304. Acesso em: 27 jul. 2020.

GRÁCIO, M. C. C.; OLIVEIRA, E. F. T. de. A inserção e o impacto internacional da pesquisa brasileira em 'estudos métricos': uma análise na base Scopus. Tendências da Pesquisa Brasileira em Ciência da Informação, João Pessoa, v. 5, n. 1, p. 1-19, 2012. Disponível em: http://hdl.handle.net/11449/114791. Acesso em: 12 fev. 2019. 
JACSO, P. As we may search: comparison of major features of the Web of Science, Scopus and Google Scholar citation-based and citation-enhanced databases. Current Science, Bengaluru, v. 89, n. 9, p. 1537-1547, nov. 2005. Disponível em: www.jstor.org/stable/24110924. Acesso em: 12 fev. 2019.

KONKIEL, S. Altmetrics: diversifying the understanding of influential scholarship. Palgrave Communications, London, v. 2, n. 16057, p. 1-7, 2016. Disponível em: https://www.nature.com/articles/palcomms201657. Acesso em: 12 fev. 2019.

LUCAS, A. et al. Indicators for the evaluation of technological activity. In: INTERNATIONAL CONFERENCE ON WWW/INTERNET AND APPLIED COMPUTING, 2017, Portugal. Proceeding... Portugal, 2017. p. 303-306. Disponível em: https://figshare.com/articles/presentation/Indicators_for_the_Evaluation_of_Technological_A ctivity/5107105/1. Acesso em: 27 jul. 2020.

MAIA, L. F. M. P. et al. ReALM: An altmetrics-based framework to map science impacts on society: a case study on Zika research. In: INTERNATIONAL CONFERENCE ON WEB INTELLIGENCE, 2019. Proceedings... Tessalônica: IEEE/WIC/ACM International Conference on Web Intelligence, 2019. p. 233-241. Disponível em: https://www.researchgate.net/publication/336660854_REALM_An_Altmetricsbased_Framework_to_Map_Science_Impacts_on_Society_A_Case_Study_on_Zika_Research . Acesso em: 27 jul. 2020.

MAIA, L. F. M. P.; LENZI, M.; OLIVEIRA, J. ReALM: An altmetrics-based web system to map science impacts on society: a case study on chikungunya research. In: SIMPÓSIO BRASILEIRO DE SISTEMAS MULTIMÍDIA E WEB (WEBMEDIA), 25., 2019, Rio de Janeiro. Anais... Porto Alegre: Sociedade Brasileira da Computação, 2019. p. 481-488. Disponível em: https://sol.sbc.org.br/index.php/webmedia/article/view/8065. Acesso em: 27 jul. 2020.

MAIA, L. F. M. P.; OLIVEIRA, J. Investigation of research impacts on the zika virus: an approach focusing on social network analysis and altmetrics. In: BRAZILIAN SYMPOSIUM ON MULTIMEDIA AND THE WEB (WEBMEDIA), 23., 2017, Gramado. Proceedings... Nova Iorque: Association for Computing Machinery, 2017. p. 413-416. Disponível em: https://www.researchgate.net/publication/320391212_Investigation_of_Research_impacts_on the_Zika_Virus_An_Approach_Focusing_on_Social_Network_Analysis_and_Altmetrics. Acesso em: 27 jul. 2020.

MAIA, M. F. S.; CAREGNATO, S. Estudos bibliométricos na comunicação científica: bibliotecas digitais como fator de revitalização. In: CONGRESSO BRASILEIRO DE CIÊNCIAS DA COMUNICAÇÃO, 27., 2004, Porto Alegre. Anais... São Paulo: INTERCOM, 2004. Disponível em: https://docplayer.com.br/4440412-Estudosbibliometricos-na-comunicacao-cientifica-bibliotecas-digitais-como-fator-de-revitalizacao1.html. Acesso em: 27 jul. 2020.

MARICATO, J. M.; LIMA, E. L.M. Impactos da altmetria: aspectos observados com análises de perfis no Facebook e Twitter. Informação e Sociedade, João Pessoa, v. 23, n. 1, p. 137145, 2017. Disponível em: 
https://periodicos.ufpb.br/ojs/index.php/ies/article/view/30921/17418. Acesso em: 27 jul. 2020.

MARICATO, J. M.; MARTINS, D. L. Altmetrics: complexities, challenges and new forms of measuring and comprehending scientific communication in the social. Biblios, Tacna, v. 27, n. 1, p. 137-145, 2017. Disponível em: https://core.ac.uk/download/pdf/194264770.pdf. Acesso em: 27 jul. 2020.

MARICATO, J. M.; VILAN FILHO, J. L. The potential for altmetrics to measure other types of impact in scientific production: academic and social impact dynamics in social media and networks. Information Research, Borås, v. 23, n. 68, p. 48-68, 2018. Disponível em: http://www.informationr.net/ir/23-1/paper780.html. Acesso em: 27 jul. 2020.

NABOUT, J. C. et al. Do traditional scientometric indicators predict social media activity on scientific knowledge? An analysis of the ecological literature. Scientometrics, Áutria, v. 115, n. 2, p. 1007-1015, 2018. Disponível em: https://link.springer.com/article/10.1007/s11192-0182678-x. Acesso em: 27 jul. 2020.

OLIVEIRA, T. M. Alternative metrics and open science in Latin America: challenges for democratization of knowledge. Transinformação, Campinas, v. 31, 2019. Disponível em: https://www.Scielo.br/Scielo.php?pid=S010337862019000100200\&script=sci_arttext\&tlng=p t. Acesso em: 27 jul. 2020.

OLIVEIRA, T. M. et al. O impacto social da ciência na área de comunicação e informação: Uma pesquisa multidimensional sobre a circulação da produção científica brasileira em sites de redes sociais. Ciência da Informação, Brasília, v. 48, n. 3, p. 285-293, 2019. Disponível em: http://revista.ibict.br/ciinf/article/view/4983. Acesso em: 27 jul. 2020.

PONTES, T. C. F.; ARAÚJO, R. F. Altmetria sobre o museu mundaneum: Estudo panorâmico sobre a difusão da produção científica nas redes sociais. Ciência da Informação, Brasília, v. 48, n. 3, p. 199-205, 2019. Disponível em:

http://revista.ibict.br/ciinf/article/view/4884. Acesso em: 27 jul. 2020.

PRIEM, J.; TARABORELLI, D.; GROTH, P.; NEYLON, C. Altmetrics: a manifesto. 2010. Disponível em: http://altmetrics.org/manifesto. Acesso em: 24 jul. 2020.

SAMPAIO, R. F.; MANCINI, M. C. Estudos de revisão sistemática: um guia para síntese criteriosa da evidência científica. Revista Brasileira de Fisioterapia, São Carlos, v. 11, n. 1, p. 83-89, set. 2007. Disponível em:

http://www.Scielo.br/Scielo.php?script=sci_arttext\&pid=S1413-

35552007000100013\&lng=en\&nrm=iso. Acesso em: 25 set. 2018.

SANTOS, P. W. Q.; ALBUQUERQUE, J. P. S. Altmetria: uma nova lente para os estudos métricos da informação. Biblionline, João Pessoa, v. 13, n. 3, p. 3-12, 2017. Disponível em: http://www.periodicos.ufpb.br/index.php/biblio/article/view/35874/19240. Acesso em: 12 dez. 2018.

SHINTAKU, M. et al. Mega-periódicos e altmetria: aproximações entre novas formas de publicação e de avaliação de impacto de resultados de pesquisa. Encontros Bibli: Revista 
Eletrônica de Biblioteconomia e Ciência da Informação, Florianópolis, v. 24, n. 54, p. 52-61, 2018. Disponível em: http://10.5007/1518-2924.2019v24n54p52. Acesso em: 22 abr. 2019.

SILVA FILHO, R. D. C.; VANZ, S. A. S. Impacto de altmetrics sobre a visibilidade de artigos em acesso aberto da enfermagem brasileira: um estudo de caso. Transinformação, Campinas, v. 31, 2019. Disponível em:

https://www.Scielo.br/Scielo.php?script=sci_arttext\&pid=S0103-37862019000100306. Acesso em: 27 jul. 2020.

SOUZA, I. V. P. Altmetria ou métricas alternativas: conceitos e principais características. AtoZ: novas práticas em informação e conhecimento, Curitiba, v. 4, n. 2, p. 58-60, 2015. Disponível em: https://revistas.ufpr.br/atoz/article/view/44554. Acesso em: 23 abr. 2019.

VALERIO, P. M.; PINHEIRO, L. V. R. Da comunicação científica à divulgação.

Transinformação, Campinas, v. 20, n. 2, p. 159-169, 2008. Disponível em: http://www.Scielo.br/pdf/tinf/v20n2/04.pdf. Acesso em: 22 mai. 2019.

VANTI, N.; SANZ-CASADO, E. Altmetria: a métrica social a serviço de uma ciência mais democrática. Transinformação, Campinas, v. 28, n. 3, p. 349-358, 2016. Disponível em: https://www.Scielo.br/Scielo.php?pid=S010337862016000300349\&script=sci_abstract\&tlng=pt. Acesso em: 27 jul. 2020.

VIEIRA, R. Q.; SANNA, M. C. O uso do estudo bibliométrico pelos pesquisadores da saúde em periódicos científicos digitais brasileiros. In: CONGRESSO BRASILEIRO DE BIBLIOTECONOMIA, DOCUMENTAÇÃO E CIÊNCIA DA INFORMAÇÃO, 25., 2013, Florianópolis. Anais... Florianópolis, 2013. Disponível em: https://portal.febab.org.br/anais/article/viewFile/1547/1548. Acesso em: 27 jul. 2020.

VOGL, S. et al. Psychology: a bibliometric analysis of psychological literature in the online media. Scientometrics, Áustria, v. 115, n. 3, p. 1253-1269, 2018. Disponível em: https://link.springer.com/article/10.1007\%2Fs11192-018-2727-5. Acesso em: 12 fev. 2019.

VOGLER, E. et al. Effektivität von altmetrics in transdisziplinären forschungsfeldern: altmetric-abdeckung von forschungsoutputs in der bildungsforschung. InformationWissenschaft und Praxis, Alemanha, v. 68, n. 2-3, p. 171-176, 2017. Disponível em: https://www.degruyter.com/view/j/iwp.2017.68.issue-2-3/iwp-2017-0035/iwp-20170035.xml. Acesso em: 12 fev. 2019. 